\title{
PHASE RECOVERY WITH NONDECAYING POTENTIALS
}

\author{
Tuncay Aktosun \\ Department of Mathematics \\ North Dakota State University \\ Fargo, ND 58105 \\ Paul E. Sacks \\ Department of Mathematics \\ Iowa State University \\ Ames, IA 50011
}

\begin{abstract}
The one-dimensional Schrödinger equation is considered when the potential is asymptotic to a positive constant on the right-half line and may possess bound states. The recovery of such a potential supported in the right-half line is studied in terms of the scattering data consisting of the magnitude of the reflection coefficient from the left, a known potential placed to the left of the unknown potential, and the magnitude of the reflection coefficient for the combined potential. It is shown that there are at most two such potentials corresponding to the scattering data, that the uniqueness holds in the absence of bound states, and that in the case of nonuniqueness the reflection coefficients of the two potentials are related to each other in a specific way. A quadrature is presented to obtain the analytic continuation of the reflection coefficient. The theory is illustrated with some explicit examples.
\end{abstract}

PACS Numbers: 03.65.Nk, 03.80.+r

Mathematics Subject Classification (1991): 34A55, 34L25, 81U40

Keywords: Phase retrieval, Inverse scattering, 1-D Schrödinger equation, Nondecaying potentials, Steplike potentials, Neutron reflectometry

Short title: Phase recovery with nondecaying potentials 


\section{INTRODUCTION}

In this paper we are concerned with the inverse problem of determining a potential in the one-dimensional Schrödinger equation from reflectivity data. This kind of measurement amounts to knowing the magnitude of the complex-valued reflection coefficient associated with the potential, meaning that the phase information is not present. Furthermore, the potential may possess bound states, and we do not assume that any information about them is explicitly available. The classical inverse scattering theory [1-3] summarized below implies that in general both the phase and bound-state data are also needed for a unique recovery of the potential, hence we will need to compensate for this missing information in some fashion. We do this by assuming that a portion of the potential, say the part to the left of the origin, is known and moreover that we can change its value and obtain reflectivity measurements as many times as we need.

This kind of data corresponds closely to that suggested in a number of recent papers on X-ray and neutron reflectometry $[4,5]$, which is thus the main motivation for our work. Essentially one seeks to determine the potential (usually a scattering length density), as a function of depth, of an unknown layered material by measuring the intensity of a beam reflected off that material as a function of the angle of incidence. The idea is then to repeat the experiment several times, with different known layers placed between the radiation source and the unknown layer, and then to use all the data to infer the phase information, the bound-state information, and subsequently the potential. In the earlier papers investigating this idea, e.g. [4,5], it was assumed that three independent measurements of reflectivity were available, but in later work $[6,7]$ it was observed that only two measurements are needed, although there is some computational advantage if three can be used. All of this work was done with the hypothesis that the potentials do not support bound states, together with other restrictions of a somewhat technical nature. These are mainly reasonable assumptions for some reflectometry applications, but 
nevertheless it is of significant interest to investigate the extent to which the analogous deductions can be made under more general assumptions on the potentials involved.

In comparison with the previous work, the essential points treated carefully here are the following:

(i) The potentials may possess bound states.

(ii) The potentials may be of exceptional type. We recall the precise definition of an exceptional potential below, but roughly it means that there is a 'zero-energy resonance' (sometimes called a half-bound state at zero energy), or equivalently that the potential is at the threshold of changing its number of bound states by one.

(iii) The potentials may have distinct limits at $\pm \infty$. This is again motivated by reflectometry applications, where such potentials arise naturally.

(iv) The potentials are allowed to have slower decay to their asymptotic limits than assumed in previous studies [8]. This is a technical point related to the zero-energy limit and the continuity of the scattering data at zero energy. Using the results in [9] we are able to overcome the technical difficulties related to the zero-energy behavior of the scattering data and hence deal with potentials decaying more slowly to their asymptotics.

Our work is a natural continuation of [10] in which we considered generic potentials without bound states, but allowing different limits at $\pm \infty$. The technical difficulties mentioned in (iv) are relevant only in the case of exceptional potentials, and hence they have not arisen in [10].

This paper is organized as follows. In Section 2 we establish our notation and recall some earlier work on the inverse scattering problem. Section 3 contains a precise statement of the problem in which we are interested, and our main results are given in Theorems 3.1, 3.2, and 3.3. These are then proved in Section 4. In Section 5 we show that one of the key 
steps in the solution process, which apparently involves analytic continuation, can actually be carried out by explicit evaluation of certain singular integrals. Finally, in Section 6 we present five examples illustrating the nonuniqueness stated in Theorems 3.1 and 3.2, the use of the data (3.1) to construct the unknown potential, the analytic continuation quadrature of Section 5, the numerical implementation of the analytic continuation quadrature, and a one-parameter family of reference potentials with which three or more reflectivity measurements add no extra information to the two-measurement data.

\section{PRELIMINARIES}

Let us proceed to give a precise formulation of the problem. We consider the onedimensional Schrödinger equation

$$
\psi^{\prime \prime}(k, x)+k^{2} \psi(k, x)=V(x) \psi(k, x), \quad x \in \mathbf{R},
$$

where the potential $V$ is real valued and satisfies

$$
V(x)-c^{2} H(x) \in L_{1}^{1}(-\infty<x<+\infty)
$$

for some $c \geq 0$. Here $H$ denotes the Heaviside function, i.e. $H(x)=1$ for $x \in \mathbf{R}^{+}$and $H(x)=0$ for $x \in \mathbf{R}^{-}$. Notice that $L_{1}^{1}$ is used in (2.2) rather than $L_{2}^{1}$, which was assumed in [8] in order to cope with certain small-energy aspects of the direct and inverse scattering problems. As mentioned in (iv) of Section 1, we never need the stronger assumption $L_{2}^{1}$ in (2.2). We use the prime to denote the derivative with respect to the spatial variable $x, \mathbf{R}^{-}:=(-\infty, 0), \mathbf{R}^{+}:=(0,+\infty)$, and $L_{q}^{1}(I)$ is the set of measurable functions $f$ on an interval $I$ such that $\int_{I} d x(1+|x|)^{q}|f(x)|$ is finite. We will use $\mathbf{C}^{+}$to denote the upper-half complex plane and $\overline{\mathbf{C}^{+}}:=\mathbf{C}^{+} \cup \mathbf{R}$.

Let

$$
\gamma:=\sqrt{k^{2}-c^{2}}
$$


where we use the branch of the square-root function with $\operatorname{Im} \gamma \geq 0$. The mapping $k \mapsto \gamma$ is analytic from $\mathbf{C}^{+}$to itself and is continuous on $\overline{\mathbf{C}^{+}}$.

The scattering states of (2.1) are those behaving like $e^{ \pm i k x}$ as $x \rightarrow-\infty$ and like $e^{ \pm i \gamma x}$ as $x \rightarrow+\infty$. Among them are the Jost solution from the left, $f_{l}(k, x)$, satisfying

$$
e^{-i \gamma x} f_{l}(k, x)=1+o(1), \quad e^{-i \gamma x} f_{l}^{\prime}(k, x)=i \gamma+o(1), \quad x \rightarrow+\infty
$$

and the Jost solution from the right, $f_{r}(k, x)$, satisfying

$$
e^{i k x} f_{r}(k, x)=1+o(1), \quad e^{i k x} f_{r}^{\prime}(k, x)=-i k+o(1), \quad x \rightarrow-\infty .
$$

The transmission and reflection coefficients from the left, $T_{l}$ and $L$, respectively, can be defined in terms of the spatial asymptotics of $f_{l}(k, x)$ as

$$
e^{-i k x} f_{l}(k, x)=\frac{1}{T_{l}(k)}+\frac{L(k)}{T_{l}(k)} e^{-2 i k x}+o(1), \quad x \rightarrow-\infty, \quad k \in \mathbf{R} \backslash\{0\}
$$

and the transmission and reflection coefficients from the right, $T_{r}$ and $R$, respectively, by using

$$
e^{i \gamma x} f_{r}(k, x)=\frac{1}{T_{r}(k)}+\frac{R(k)}{T_{r}(k)} e^{2 i \gamma x}+o(1), \quad x \rightarrow+\infty, \quad k \in \mathbf{R} \backslash[-c, c] .
$$

If (2.2) holds, then for each $x \in \mathbf{R}$ the Jost solutions are known [8,11] to be analytic in $k \in \mathbf{C}^{+}$and continuous in $k \in \overline{\mathbf{C}^{+}}$.

A distinction which is important for some aspects of the theory is the relationship between $f_{l}$ and $f_{r}$ at $k=0$. If $f_{l}(0, x)$ and $f_{r}(0, x)$ are linearly independent on $\mathbf{R}$, we say that the potential $V$ is generic and otherwise $V$ is exceptional. Thus, $V$ is exceptional if and only if (2.1) with $k=0$ has a solution which is bounded on $\mathbf{R}$.

It is known that $L$ can be extended from $k \in \mathbf{R} \backslash\{0\}$ to $k \in \mathbf{R}, R$ from $k \in \mathbf{R} \backslash[-c, c]$ to $k \in \mathbf{R}$; moreover, $T_{l}$ has a meromorphic extension from $k \in \mathbf{R} \backslash\{0\}$ to $k \in \overline{\mathbf{C}^{+}}$, and $T_{r}$ 
from $k \in \mathbf{R} \backslash\{0\}$ to $k \in \overline{\mathbf{C}^{+}}$in the generic case and to $k \in \overline{\mathbf{C}^{+}} \backslash\{0\}$ in the exceptional case. Furthermore, the following are known to hold:

$$
\begin{gathered}
1-|L(k)|^{2}=\frac{\gamma}{k}\left|T_{l}(k)\right|^{2} \neq 0, \quad k \in \mathbf{R} \backslash[-c, c], \\
L(k)=\frac{T_{l}(k)}{T_{l}^{*}(k)}, \quad k \in[-c, c], \\
R(k)=-\frac{L(k)^{*} T_{l}(k)}{T_{l}(k)^{*}}, \quad k \in \mathbf{R} ; \quad T_{r}(k)=\frac{\gamma}{k} T_{l}(k), \quad k \in \overline{\mathbf{C}^{+}} \backslash\{0\}, \\
T_{l}(-k)=T_{l}(k)^{*}, \quad L(-k)=L(k)^{*}, \quad R(-k)=R(k)^{*}, \quad k \in \mathbf{R},
\end{gathered}
$$

where the asterisk denotes complex conjugation. From (2.6) and (2.7) we see that

$$
c=\sup _{k \geq 0}\{k:|L(t)|=1,0 \leq t \leq k\} .
$$

For the validity of $(2.7),(2.8)$, and the continuity of $T_{l}$ in the exceptional case at $k=0$ when (2.2) is satisfied, the reader is referred to [9]; otherwise, these and other properties of the scattering coefficients are available in $[8,11]$. As seen from $(2.8), T_{r}$ is a simple multiple of $T_{l}$, and hence for simplicity, in the rest of the paper we will drop the subscript $l$ and use $T$ to denote the transmission coefficient from the left.

Next, the bound states of $V$ correspond to the square-integrable solutions of (2.1), and such states can occur $[8,11]$ only at certain values $k=i \kappa_{j}$ on the positive imaginary axis, which are exactly the poles of $T$. When $V$ satisfies $(2.2)$, it follows from $[8,9,11]$ that the number of bound states is finite, which we will denote by $N$. For each bound state there correspond the norming constants $\rho_{j}$ and $\xi_{j}$ defined by

$$
\frac{1}{\rho_{j}}:=\int_{-\infty}^{\infty} d y f_{r}\left(i \kappa_{j}, y\right)^{2}, \quad \frac{1}{\xi_{j}}:=\int_{-\infty}^{\infty} d y f_{l}\left(i \kappa_{j}, y\right)^{2} .
$$

Collectively, $\kappa_{j}, \rho_{j}$, and $\xi_{j}$ constitute the bound-state data; the transmission and reflection coefficients plus the bound-state data make up the scattering data for $V$. 
The direct scattering problem for (2.1) is to determine the scattering coefficients and bound states when the potential is known, whereas the inverse scattering problem is to recover the potential from the scattering data or more likely from a certain subset of the scattering data. Such direct and inverse scattering problems were analyzed first by Buslaev and Fomin [11] and elaborated later by Legendre [12] and Cohen and Kappeler [8]. Roughly speaking, $V$ is uniquely determined by solving one of the two Marchenko integral equations $[8,11]$ using the data

$$
\{L(k), 0<k<+\infty\}, \quad\left\{\kappa_{j}\right\}_{j=1}^{N} \quad\left\{\rho_{j}\right\}_{j=1}^{N},
$$

or

$$
\{R(k), c<k<+\infty\}, \quad\{|T(k)|, 0<k<c\}, \quad\left\{\kappa_{j}\right\}_{j=1}^{N} \quad\left\{\xi_{j}\right\}_{j=1}^{N} .
$$

The precise results are as follows: If $V$ is generic and satisfies (2.2), then the unique recovery was proved in [11]. For generic potentials or exceptional potentials satisfying (2.2) but $L_{1}^{1}$ replaced with $L_{2}^{1}$, the uniqueness was proved in [8]. For an exceptional $V$ satisfying (2.2), the uniqueness also follows from [8] provided the following additional condition holds:

$$
\lim _{k \rightarrow 0} \frac{W(k)}{k}=i \alpha, \quad \text { for some } \alpha \in \mathbf{R} \backslash\{0\},
$$

where $W(k):=f_{l} f_{r}^{\prime}-f_{l}^{\prime} f_{r}$ denotes the Wronskian of $f_{l}$ and $f_{r}$ and is equal to $-2 i k / T(k)$. In [9] it has been shown that (2.13) holds for all exceptional potentials satisfying (2.2); thus, either of the data sets (2.11) and (2.12) is sufficient to uniquely determine any potential satisfying (2.2). For the classical case $c=0$, the literature is of course much larger, see e.g. $[1,2,3,13,14]$.

\section{MAIN THEOREMS}

Let us fragment $V$ as $V=V_{1}+V_{2}$, where $V_{1}$ has support in $\mathbf{R}^{-}$and $V_{2}$ has support in $\mathbf{R}^{+}$. As seen from (2.2) we have $V_{1} \in L_{1}^{1}\left(\mathbf{R}^{-}\right)$and $V_{2}-c^{2} \in L_{1}^{1}\left(\mathbf{R}^{+}\right)$. The problem which 
will be studied in this paper is the following: Determine $V_{2}$ for all $x>0$ given the data

$$
\{|L(k)|, 0<k<+\infty\}, \quad\left\{\left|L_{2}(k)\right|, 0<k<+\infty\right\}, \quad\left\{V_{1}(x),-\infty<x<0\right\} .
$$

Here $L_{2}$ denotes reflection coefficient from the left for $V_{2}$. Thus, we are missing both the bound-state and phase information, but we compensate by assuming that $V$ is known for $x<0$ and also that the magnitude of the reflection coefficient is known both for the complete potential $V$ and for its right half $V_{2}$. Clearly, we must assume $V_{1} \not \equiv 0$ so that $L_{2} \not \equiv L$.

In the case of decaying potentials, i.e. when $c=0$, assuming that no bound states exist and that $V, V_{1}$, and $V_{2}$ are all generic, in order to recover $V_{2}$ the 'three-measurement method' $[4,5,15,16]$ uses an additional set of reflectivity besides the data (3.1). The exact conditions for the validity of this method have been given in [10]; in Section 6 we present an explicit example, applicable whether $c=0$ or $c>0$, showing that without those conditions any number of extra reflectivity measurements will add no additional information to the two-measurement data (3.1). Nevertheless, even if the three-measurement method fails [10], the data (3.1) determine $V_{2}$ uniquely and the recovery can be achieved by the 'twomeasurement' method $[6,10]$.

The case $c>0$ was considered in [10], but still with the assumption of generic potentials and no bound states, and the two-measurement method has been extended [10] to the case $c>0$. Let us also mention the experimental study [17] using the three-measurement data and the paper [7] relying on the data consisting of two known constant potentials placed behind a compactly supported unknown sample; the method of [7] can be extended to more general situations [10].

Under the less restrictive assumptions, i.e. by allowing exceptional potentials, bound states, positive $c$, and potentials satisfying $L_{1}^{1}$ in $(2.2)$ rather than $L_{2}^{1}$, we obtain the following results. 
Theorem 3.1 Assume that $V:=V_{1}+V_{2}$ is real valued and satisfies (2.2) for some $c \geq 0$. If $V_{1} \not \equiv 0$, then there are at most two distinct potentials as $V_{2}$ having the data (3.1) in common. In general the uniqueness does not hold.

In Section 6 we will illustrate the nonuniqueness stated in the above theorem by an explicit example. The next result shows that the uniqueness is still in some sense typical.

Theorem 3.2 If there exist two distinct potentials as $V_{2}$ stated in Theorem 3.1, both corresponding to the same data (3.1), then $1-R_{1} L_{2}$ must be a certain rational function of $k$ whose zeros and poles can occur only on the imaginary axis.

Finally, when bound states are not allowed we always have the uniqueness.

Theorem 3.3 Assume $V:=V_{1}+V_{2}$ is real valued and satisfies (2.2) for some $c \geq 0$. If $V_{1} \not \equiv 0$ and neither $V_{1}$ nor $V_{2}$ has any bound states, then $V_{2}$ is uniquely determined by the data (3.1).

\section{PROOFS OF THE MAIN THEOREMS}

In this section we present the proofs of Theorems 3.1, 3.2, and 3.3. In preparation we give two propositions, whose proofs are fairly straightforward modifications of arguments which have appeared elsewhere.

Proposition 4.1 Assume that $V$ is real valued, it has support in $\mathbf{R}^{+}$, and $V-c^{2} \in L_{1}^{1}\left(\mathbf{R}^{+}\right)$ for some $c \geq 0$. Then $L$ has an extension from $\mathbf{R} \backslash\{0\}$ which is continuous in $\overline{\mathbf{C}^{+}}$and analytic in $\mathbf{C}^{+}$except at a finite number of simple poles on the positive imaginary axis which exactly correspond to the bound states of $V$. Similarly, if $V$ has support in $\mathbf{R}^{-}$and $V-c^{2} \in L_{1}^{1}\left(\mathbf{R}^{-}\right)$for some $c \geq 0$, then $R$ has an extension from $\mathbf{R} \backslash\{0\}$ which is continuous in $\overline{\mathbf{C}^{+}}$and analytic in $\mathbf{C}^{+}$except at a finite number of poles on the positive imaginary axis which exactly correspond to the bound states of $V$.

PROOF: The two cases are similar, so we only prove the first one. As in the case $c=0$, one 
knows $[8,11]$ that $T$ is analytic in $\mathbf{C}^{+}$and continuous in $\overline{\mathbf{C}^{+}}$except for a finite number of simple poles $k=i \kappa_{1}, \ldots, i \kappa_{N}$ all located on the positive imaginary axis and corresponding to the bound states of $V$. The continuity at $k=0$ (which in turn implies the finiteness of the number of poles) is actually a somewhat delicate point proved only for generic potentials satisfying (2.2) in [11] and only for exceptional potentials satisfying the stronger condition with $L_{2}^{1}$ in (2.2) instead of $L_{1}^{1}$ in [8]. The proof for exceptional potentials satisfying (2.2) is rather technical and remote from the main purpose of our paper, and thus we refer the reader to [9] for details.

Based on the linear independence of $f_{r}$ and $f_{r}^{*}$, as in (4.8+) of [8], we have

$$
T(k) f_{l}(k, x)=f_{r}^{*}(k, x)+L(k) f_{r}(k, x), \quad x \in \mathbf{R}, \quad k \in \mathbf{R} \backslash\{0\},
$$

and hence, since $f_{r}(k, x)=e^{-i k x}$ for $x \leq 0$, we get

$$
L(k)=T(k) f_{l}(k, x) e^{i k x}-e^{2 i k x}, \quad x \in \mathbf{R}, \quad k \in \mathbf{R} \backslash\{0\} .
$$

It is known $[8,11]$ that $f_{l}(\cdot, x)$ is analytic in $\mathbf{C}^{+}$and continuous in $\overline{\mathbf{C}^{+}}$; moreover, $f_{l}$ cannot vanish for all $x \leq 0$. Thus, (4.1) can be extended from $k \in \mathbf{R} \backslash\{0\}$ to $\overline{\mathbf{C}^{+}}$and the analyticity and continuity properties of $L$ coincide with those of $T$.

Proposition 4.2 Assume that $V$ is real valued, it has support in $\mathbf{R}^{+}$, and that $V-c^{2} \in$ $L_{1}^{1}\left(\mathbf{R}^{+}\right)$for some $c \geq 0$. Then $V$ is uniquely determined by $\left\{L(k): k_{1}<k<k_{2}\right\}$ for any nonempty interval $\left(k_{1}, k_{2}\right) \subset \mathbf{R}$.

PROOF: The argument we give here is essentially the same as in [18]; a somewhat different proof can also be given as in [19].

It is enough to prove that the quantities in (2.11) are determined by $\left\{L(k), k_{1}<k<\right.$ $\left.k_{2}\right\}$. By Proposition 4.1, $L$ has an extension from $k \in\left(k_{1}, k_{2}\right)$ to $\overline{\mathbf{C}^{+}}$with simple poles corresponding to the bound states $k=i \kappa_{j}$ of $V$. Thus, we only need to show that $\rho_{j}$ in (2.11) is determined by $L$. We claim that $\operatorname{Res}\left(L, i \kappa_{j}\right)$, the residue of $L$ at $i \kappa_{j}$, is equal to 
$i \rho_{j}$. We can see this as follows. Let

$$
\mu_{j}:=\frac{f_{l}\left(i \kappa_{j}, x\right)}{f_{r}\left(i \kappa_{j}, x\right)}, \quad x \in \mathbf{R},
$$

which is a nonzero constant since $f_{l}$ and $f_{r}$ are linearly dependent at a bound state. The residue of $T$ at $i \kappa_{j}$ is nonzero and found from [11]

$$
\frac{1}{\operatorname{Res}\left(T, i \kappa_{j}\right)}=-i \int_{-\infty}^{\infty} d y f_{l}\left(i \kappa_{j}, y\right) f_{r}\left(i \kappa_{j}, y\right)
$$

and by (4.1) we have

$$
\operatorname{Res}\left(L, i \kappa_{j}\right)=e^{-\kappa_{j} x} f_{l}\left(i \kappa_{j}, x\right) \operatorname{Res}\left(T, i \kappa_{j}\right), \quad x \leq 0 .
$$

Thus, using (4.2) and (4.3) in (4.4) we get

$$
\operatorname{Res}\left(L, i \kappa_{j}\right)=\frac{i e^{-\kappa_{j} x} \mu_{j} f_{r}\left(i \kappa_{j}, x\right)}{\mu_{j} \int_{-\infty}^{\infty} d y f_{r}\left(i \kappa_{j}, y\right)^{2}}, \quad x \leq 0 .
$$

From (4.5), using (2.10) and the fact that $f_{r}\left(i \kappa_{j}, x\right)=e^{\kappa_{j} x}$ for $x \leq 0$, we get $\operatorname{Res}\left(L, i \kappa_{j}\right)=$ $i \rho_{j}$.

Next we give the proofs of Theorems 3.1, 3.2, and 3.3.

Proof of Theorem 3.1: Let $T_{1}, T_{2}$, and $T$ denote the transmission coefficients from the left for $V_{1}, V_{2}$, and $V$, respectively. If $V$ satisfies (2.2) for some $c \geq 0$, then it is known (see e.g. $[9,10])$ that

$$
\frac{T_{1} T_{2}}{T}=1-R_{1} L_{2}, \quad k \in \mathbf{R},
$$

where $R_{1}$ is the reflection coefficient from the right for $V_{1}$ and $L_{2}$ is the reflection coefficient from the left for $V_{2}$.

On account of (2.6), (2.9), and knowledge of $V_{1}$, the quantities $|T|,\left|T_{1}\right|,\left|T_{2}\right|$, and $\left|R_{1}\right|$ are known for $k \in \mathbf{R} \backslash[-c, c]$ from our data (3.1); then $\left|R_{1} L_{2}\right|$ and, via (4.6), $\left|1-R_{1} L_{2}\right|$ 
are uniquely determined for any fixed $k \in \mathbf{R} \backslash[-c, c]$. In fact, the real part of $R_{1} L_{2}$ for $k \in \mathbf{R} \backslash[-c, c]$ can be written explicitly (see e.g. [10]) as $\operatorname{Re}\left\{R_{1}(k) L_{2}(k)\right\}=A(k)$, where

$$
A(k):==\frac{1}{2}\left[1+\left|R_{1}(k)\right|^{2}\left|L_{2}(k)\right|^{2}-\frac{\left(1-\left|R_{1}(k)\right|^{2}\right)\left(1-\left|L_{2}(k)\right|^{2}\right)}{1-|L(k)|^{2}}\right] .
$$

Thus, for each fixed $k \in \mathbf{R} \backslash[-c, c]$, the imaginary part of $R_{1}(k) L_{2}(k)$ determined up to a sign.

We now claim that the set $\left\{k \in \mathbf{R}: R_{1}(k)=0\right\}$ must be of measure zero. From Corollary to Theorem 4.3 of [2] it follows that the potential $V_{1}^{[0]}$ obtained from $V_{1}$ by removing all its bound states is also supported in $\mathbf{R}^{-}$, and hence the corresponding reflection coefficient $R^{[0]}$ is analytic in $\mathbf{C}^{+}$and belongs to the Hardy space $H_{+}^{2}$; it is known [20] that if such a function vanishes on a set of positive measure, then it must be identically zero. Since $\left|R_{1}^{[0]}\right| \equiv\left|R_{1}\right|$ on $\mathbf{R}$, it would then follow that $R_{1} \equiv 0$ on $\mathbf{R}$, contradicting our assumption $V_{1} \not \equiv 0$.

Since the quantity $R_{1}(k) L_{2}(k)$ for each fixed $k \in \mathbf{R} \backslash[-c, c]$ is determined up to a sign in its imaginary part and $R_{1}(k) \neq 0$ a.e., it follows that for almost every $k \in \mathbf{R} \backslash[-c, c]$, there are at most two choices of $L_{2}(k)$ consistent with the given scattering data, namely $[1-F(k)] / R_{1}(k)$ and $\left[1-F^{*}(k)\right] / R_{1}(k)$, where $F$ is the quantity defined as

$$
F(k):=1-R_{1}(k) L_{2}(k)
$$

If we pick any point $k_{0}>c$ at which $\operatorname{Im}\left\{F\left(k_{0}\right)\right\} \neq 0$, then on some interval $\left(k_{1}, k_{2}\right)$ containing $k_{0}$, exactly two continuous functions as the possible choices for $L_{2}$, are uniquely determined, which subsequently determine two possibilities for $V_{2}$, according to Proposition 4.2. By the uniqueness of analytic continuation, any other such choice of $k_{0}$ must lead to the same two possibilities for $V_{2}$. Example 6.1 shows that it is possible to have two distinct potentials as $V_{2}$ corresponding to the data (3.1).

Proof of Theorem 3.2: Now suppose $\tilde{V}_{2}$ is the potential distinct from $V_{2}$ and supported in $\mathbf{R}^{+}$and $\tilde{V}_{2}-c^{2} \in L_{1}^{1}(\mathbf{R})$. Set $\tilde{V}:=V_{1}+\tilde{V}_{2}$ and let $\tilde{L}$ and $\tilde{L}_{2}$ denote the reflection coefficients 
from the left for $\tilde{V}$ and $\tilde{V}_{2}$, respectively. Thus we are assuming that $|L| \equiv|\tilde{L}|,\left|L_{2}\right| \equiv\left|\tilde{L}_{2}\right|$, and $V_{2} \not \equiv \tilde{V}_{2}$. Defining $F:=1-R_{1} L_{2}$ and $\tilde{F}:=1-R_{1} \tilde{L}_{2}$, from the proof of Theorem 3.1 it follows that $\tilde{F} \equiv F^{*}$ for $k \in \mathbf{R}$. Hence, by (4.6) and Proposition 4.1, it follows that $F$ has a meromorphic extension to the whole complex plane $\mathbf{C}$.

Let $k=i \beta$ denote the set of bound states of $V$, and similarly let us use $\beta_{1}, \beta_{2}, \tilde{\beta}$, $\tilde{\beta}_{2}$ to denote the bound states of $V_{1}, V_{2}, \tilde{V}, \tilde{V}_{2}$, respectively. Let $N, \tilde{N}, N_{1}, N_{2}, \tilde{N}_{2}$ denote the number of bound states of $V, \tilde{V}, V_{1}, V_{2}, \tilde{V}_{2}$, respectively. The finiteness of these numbers is assured [9] for the class of potentials satisfying (2.2). Let us use $(k-i \beta)$ to denote the product of such factors, i.e. if $\beta$ contains the elements $\beta^{(1)}, \cdots, \beta^{(N)}$, then $(k-i \beta):=\prod_{j=1}^{N}\left(k-i \beta^{(j)}\right)$. We use similar notations $\left(k-i \beta_{1}\right),\left(k-i \beta_{2}\right),(k-i \tilde{\beta})$, and $\left(k-i \tilde{\beta}_{2}\right)$. Let us separate the poles of $F$ and $\tilde{F}$ by defining

$$
F_{0}:=F \frac{k-i \beta_{1}}{k+i \beta_{1}} \frac{k-i \beta_{2}}{k+i \beta_{2}}, \quad \tilde{F}_{0}:=\tilde{F} \frac{k-i \beta_{1}}{k+i \beta_{1}} \frac{k-i \tilde{\beta}_{2}}{k+i \tilde{\beta}_{2}}
$$

and hence $F_{0}$ and $\tilde{F}_{0}$ are analytic in $\mathbf{C}^{+}$, are continuous in $\overline{\mathbf{C}^{+}}$, and $1+O(1 / k)$ as $k \rightarrow \infty$ in $\overline{\mathbf{C}^{+}}$. From $\tilde{F}=F^{*}$, we get

$$
F_{0} \frac{k+i \beta_{1}}{k-i \beta_{1}} \frac{k+i \beta_{2}}{k-i \beta_{2}}=\tilde{F}_{0}^{*} \frac{k-i \beta_{1}}{k+i \beta_{1}} \frac{k-i \tilde{\beta}_{2}}{k+i \tilde{\beta}_{2}}, \quad k \in \mathbf{R},
$$

or equivalently

$$
F_{0}\left(k+i \beta_{1}\right)^{2}\left(k+i \beta_{2}\right)\left(k+i \tilde{\beta}_{2}\right)=\tilde{F}_{0}^{*}\left(k-i \beta_{1}\right)^{2}\left(k-i \beta_{2}\right)\left(k-i \tilde{\beta}_{2}\right), \quad k \in \mathbf{R} .
$$

In (4.9) the left- and right-hand sides are analytic in the upper- and lower-half planes respectively, continuous in the respective closed half planes, and agree on the real axis. Hence either side extends to an entire function which moreover satisfies a polynomial growth condition at infinity, and so is a polynomial of degree $2 N_{1}+N_{2}+\tilde{N}_{2}$, which we denote by $P(k)$. Thus,

$$
F_{0}=\frac{P}{\left(k+i \beta_{1}\right)^{2}\left(k+i \beta_{2}\right)\left(k+i \tilde{\beta}_{2}\right)}, \quad \tilde{F}_{0}=\frac{P^{*}}{\left(k+i \beta_{1}\right)^{2}\left(k+i \beta_{2}\right)\left(k+i \tilde{\beta}_{2}\right)} .
$$


From (4.6) we see that $F=T_{1} T_{2} / T$ and $\tilde{F}=T_{1} \tilde{T}_{2} / T$, and hence the zeros of $F$ and $\tilde{F}$ on the positive imaginary axis are related to the poles of $T$ and $\tilde{T}$. In fact, using the results in Section 7 of [9] it follows that

$$
F=\frac{k^{j}(k-i \beta)(k+i \tilde{\beta})}{\left(k+i \beta_{1}\right)\left(k-i \beta_{1}\right)\left(k-i \beta_{2}\right)\left(k+i \tilde{\beta}_{2}\right)}, \quad k \in \mathbf{C},
$$

where $j=0$ if $V_{1}$ and $V_{2}$ are both exceptional, $j=2$ if $V_{1}$ and $V_{2}$ are generic and $V$ is exceptional, and $j=1$ in all the other cases. Thus, in the case of nonuniqueness, $R_{1} L_{2}$ is a rational function in $\mathbf{C}$ and $1-R_{1} L_{2}$ is given by (4.10).

Proof of Theorem 3.3: We need to take into consideration that some or all of $V, V_{1}$, and $V_{2}$ may be exceptional. However, the proof of Theorem 4.2 of [10] still verbatim applies here provided that $R_{1} L_{2}$ is continuous at $k=0$. This continuity was proved in [9] for exceptional potentials satisfying (2.2).

\section{ANALYTIC CONTINUATION}

As we have seen in Theorems 3.1-3.3 and their proofs, our reflectivity data (3.1) determine $\left\{L_{2}(k), k \in \mathbf{R} \backslash[-c, c]\right\}$ up to at most two candidates, namely as $(1-F) / R_{1}$ and $\left(1-F^{*}\right) / R_{1}$ with

$$
\operatorname{Re}\{F(k)\}=1-A(k), \quad[\operatorname{Im}\{F(k)\}]^{2}=\frac{1}{4} Z(k), \quad k \in \mathbf{R} \backslash[-c, c],
$$

where $A(k)$ is the quantity defined in (4.7) and

$$
Z(k):=4\left|R_{1}(k)\right|^{2}\left|L_{2}(k)\right|^{2}-4 A(k)^{2}, \quad k \in \mathbf{R} \backslash[-c, c] .
$$

It is known [10] that $Z(k) \geq 0$ for $k \in \mathbf{R} \backslash[-c, c]$.

Now assume that we are somehow able to pick the 'correct' $\left\{L_{2}(k), k \in \mathbf{R} \backslash[-c, c]\right\}$ among the two candidates, and we would like to construct $V_{2}$ by using the Marchenko method $[8,11]$ with $(2.11)$ or $(2.12)$ as the input data. When $c>0$ it is apparent that some 
sort of analytic continuation step is needed to carry out the reconstruction procedure. For example, to obtain the data (2.11), we need to find $\left\{L_{2}(k), k \in[-c, c]\right\}$, and when there are bound states, we also need to find the poles of $L_{2}(k)$ on the positive imaginary axis and, as indicated in the proof of Proposition 4.2, the residues of $L_{2}$ at such poles. Assuming that the poles of $L_{2}$ are known (by the uniqueness of analytic continuation, these poles are uniquely determined even though there are no exact methods for such a determination), in this section we will explain how $T_{2}$ and $L_{2}$ can be constructed for $k \in \overline{\mathbf{C}^{+}}$by evaluation of certain explicit singular integrals. We will present the theory first when there are no bound states, and at the end of the section we will describe the modifications in the presence of bound states.

The mapping $\gamma \mapsto k$, the inverse of the mapping $k \mapsto \gamma$ given in (2.3), is analytic in $\gamma \in \mathbf{C}^{+} \backslash i(0, c]$ and is continuous in $\gamma \in \overline{\mathbf{C}^{+}} \backslash i[0, c)$. We will denote this inverse map by $k(\gamma)$.

Thus, we assume that we know $\left\{L_{2}(k), c<k<+\infty\right\}$ and, because of (2.3) and (2.9), this amounts to knowing $\left\{L_{2}(k(\gamma)), \gamma \in \mathbf{R}\right\}$. Since $V_{2} \equiv 0$ for $x<0$, from (2.5) and Proposition 4.1 we have

$$
f_{l ; 2}(k, 0)=\frac{1+L_{2}(k)}{T_{2}(k)}, \quad f_{l ; 2}^{\prime}(k, 0)=i k \frac{1-L_{2}(k)}{T_{2}(k)}, \quad k \in \overline{\mathbf{C}^{+}},
$$

where $f_{l ; 2}(k, x)$ denotes the Jost solution from the left for $V_{2}$, and $L_{2}$ and $T_{2}$ denote the corresponding reflection and transmission coefficients from the left. From (5.3) we get

$$
\begin{array}{ll}
L_{2}(k)=\frac{i k f_{l ; 2}(k, 0)-f_{l ; 2}^{\prime}(k, 0)}{i k f_{l ; 2}(k, 0)+f_{l ; 2}^{\prime}(k, 0)}, & k \in \overline{\mathbf{C}^{+}}, \\
T_{2}(k)=\frac{2 i k}{i k f_{l ; 2}(k, 0)+f_{l ; 2}^{\prime}(k, 0)}, & k \in \overline{\mathbf{C}^{+}} .
\end{array}
$$

It follows that it is enough to obtain appropriate expressions for $f_{l ; 2}(k, 0)$ and $f_{l ; 2}^{\prime}(k, 0)$ for $k \in \overline{\mathbf{C}^{+}}$. This is accomplished by defining

$$
X(\gamma):=i k(\gamma) \frac{1-L_{2}(k(\gamma))}{1+L_{2}(k(\gamma))}-i \gamma, \quad \gamma \in \mathbf{R}
$$


and by using the following result.

Theorem 5.1 Assume that $V_{2}$ is real valued, it has support in $\mathbf{R}^{+}$, it is free of bound states, and $V_{2}-c^{2} \in L_{1}^{1}\left(\mathbf{R}^{+}\right)$. Then, we have

$$
\begin{gathered}
f_{l ; 2}(k(\gamma), 0)=\exp \left(\frac{1}{2 \pi i} \int_{-\infty}^{\infty} d t \frac{\log B(t)}{t-\gamma-i 0^{+}}\right), \quad \gamma \in \overline{\mathbf{C}^{+}}, \\
f_{l ; 2}^{\prime}(k(\gamma), 0)=i(\gamma+i) \exp \left(\frac{1}{2 \pi i} \int_{-\infty}^{\infty} d t \frac{\log E(t)}{t-\gamma-i 0^{+}}\right), \quad \gamma \in \overline{\mathbf{C}^{+}}
\end{gathered}
$$

where

$$
B(t):=\frac{t}{t+\operatorname{Im}\{X(t)\}}, \quad E(t):=\frac{t\left[t^{2}+2 t \operatorname{Im}\{X(t)\}+|X(t)|^{2}\right]}{\left(t^{2}+1\right)[t+\operatorname{Im}\{X(t)\}]}
$$

Moreover, $\operatorname{Im}\{X(t)\}$ is an odd function of $t \in \mathbf{R}$. Hence, $B(t)$ and $E(t)$ are even functions of $t \in \mathbf{R}$, and (5.7) and (5.8) can also be written as

$$
\begin{gathered}
f_{l ; 2}(k(\gamma), 0)=\exp \left(\frac{\gamma}{\pi i} \int_{0}^{\infty} d t \frac{\log B(t)}{t^{2}-\left(\gamma+i 0^{+}\right)^{2}}\right), \quad \gamma \in \overline{\mathbf{C}^{+}} \\
f_{l ; 2}^{\prime}(k(\gamma), 0)=i(\gamma+i) \exp \left(\frac{\gamma}{\pi i} \int_{0}^{\infty} d t \frac{\log E(t)}{t^{2}-\left(\gamma+i 0^{+}\right)^{2}}\right), \quad \gamma \in \overline{\mathbf{C}^{+}}
\end{gathered}
$$

Furthermore, $B(t)$ and $E(t)$ are both $1+o(1 / t)$ as $t \rightarrow+\infty$, and the integrands in (5.10) and (5.11) are $o\left(1 / t^{3}\right)$ as $t \rightarrow+\infty$.

The proof of (5.7) will be given in Proposition 5.5, that of (5.8) in Proposition 5.6, and the oddness property of $\operatorname{Im}\{X(t)\}$ will be proved in Proposition 5.4. The behavior of $B(t)$, $E(t)$, and the integrands in (5.10) and (5.11) as $t \rightarrow+\infty$ will be proved in Proposition 5.7.

By using (5.10) and (5.11) at points $\gamma=i \omega$ for $0<\omega<c$, we can obtain $f_{l ; 2}(k, 0)$ and $f_{l ; 2}^{\prime}(k, 0)$ and subsequently $L_{2}(k)$ for $k \in[-c, c]$; this is also fairly easy to implement numerically and is illustrated in Example 6.5. Since the integrands in (5.10) and (5.11) are $o\left(1 / t^{3}\right)$ as $t \rightarrow+\infty$, there is not much difficulty in numerically evaluating the integrals 
over an infinite interval. The only instability may be expected as $t \rightarrow 0^{+}$if one tries to evaluate the integrals in (5.10) and (5.11) at $\gamma=0$.

At the end of this section, we will outline how the procedure of the construction of $L_{2}(k)$ and $T_{2}(k)$ for $k \in \overline{\mathbf{C}^{+}}$from $\left\{L_{2}(k(\gamma)), \gamma \in \mathbf{R}\right\}$ can be carried when there are bound states.

Proposition 5.2 Assume that $g$ is an analytic function in $\gamma \in \mathbf{C}^{+}$and continuous in $\gamma \in \overline{\mathbf{C}^{+}}$and that

(i) $g(\gamma) \neq 0$ in $\overline{\mathbf{C}^{+}} \backslash\{0\}$, and $\gamma=0$ is either not a zero of $g$ or a zero of finite multiplicity.

(ii) $g(-\gamma)=g(\gamma)^{*}$ for $\gamma \in \mathbf{R}$.

(iii) $g(\gamma) \rightarrow 1$ uniformly as $\gamma \rightarrow \infty$ in $\mathbf{C}^{+}$.

Then $g$ has the representation

$$
g(\gamma)=\exp \left(\frac{1}{2 \pi i} \int_{-\infty}^{\infty} d t \frac{\log |g(t)|^{2}}{t-\gamma-i 0^{+}}\right), \quad \gamma \in \overline{\mathbf{C}^{+}}
$$

PROOF: Let log denote a branch of the (complex) logarithm function such that $\log g(z)$ is analytic in $z \in \mathbf{C}^{+}$and continuous in $z \in \overline{\mathbf{C}^{+}}$and $\log g(z) \rightarrow 0$ as $z \rightarrow \infty$ in $\overline{\mathbf{C}^{+}}$. Then, by the Cauchy integral formula we have

$$
\begin{gathered}
\log g(\gamma)=\frac{1}{2 \pi i} \int_{-\infty}^{\infty} d t \frac{\log g(t)}{t-\gamma}, \quad \gamma \in \mathbf{C}^{+} \\
0=\frac{1}{2 \pi i} \int_{-\infty}^{\infty} d t \frac{\log g(-t)}{t-\gamma}, \quad \gamma \in \mathbf{C}^{+} .
\end{gathered}
$$

A finite-multiplicity zero of $g$ at $t=0$ does not affect these two integral identities because the integral of $[\log g(t)] /(t \pm \gamma)$ along the upper semicircle centered at the origin vanishes as the radius of that semicircle approaches zero. Adding these two identities and using (ii), we obtain (5.12), where we see that the values for $\gamma \in \mathbf{R}$ are obtained by letting $\operatorname{Im} \gamma \rightarrow 0^{+}$. 
We will apply $(5.12)$ to $g(\gamma)=f_{l ; 2}(k(\gamma), 0)$ and $g(\gamma)=\frac{1}{i(\gamma+i)} f_{l ; 2}^{\prime}(k(\gamma), 0)$ to obtain (5.7) and (5.8), respectively. So, we need to show that these two functions satisfy the hypotheses of Proposition 5.2.

Proposition 5.3 Assume that $V_{2}$ is real valued, it has support in $\mathbf{R}^{+}$, it is free of bound states, and $V_{2}-c^{2} \in L_{1}^{1}\left(\mathbf{R}^{+}\right)$. Then, neither $f_{l ; 2}(k, 0)$ nor $f_{l ; 2}^{\prime}(k, 0)$ can vanish when $k \in \overline{\mathbf{C}^{+}} \backslash[-c, c]$. Moreover, $f_{l ; 2}( \pm c, 0) \neq 0$ if $L_{2}( \pm c) \neq-1$, and $f_{l ; 2}(k, 0)$ has a simple zero at $k= \pm c$ if $L_{2}( \pm c)=-1$. Similarly, $f_{l ; 2}^{\prime}( \pm c, 0) \neq 0$ if $L_{2}( \pm c) \neq 1$, and $f_{l ; 2}^{\prime}(k, 0)$ has a simple zero at $k= \pm c$ if $L_{2}( \pm c)=1$.

PROOF: By (5.3) we see that neither $f_{l ; 2}(k, 0)$ nor $f_{l ; 2}^{\prime}(k, 0)$ has any zeros in $\overline{\mathbf{C}^{+}} \backslash\{0\}$ provided $L_{2}(k) \neq \pm 1$ there. The analytic extension of $L_{2}(k)$ vanishes as $k \rightarrow \infty$ in $\overline{\mathbf{C}^{+}}$, $\left|L_{2}(k)\right|<1$ for $k \in \mathbf{R} \backslash[-c, c]$ as shown in (2.6), and $\left|L_{2}(k)\right|=1$ for $k \in[-c, c]$ because of (2.7). Thus, by the maximum modulus principle we get $\left|L_{2}(k)\right|<1$ for $k \in \mathbf{C}^{+}$and hence for $k \in \overline{\mathbf{C}^{+}} \backslash[-c, c]$. The behavior of $f_{l ; 2}( \pm c, 0)$ and $f_{l ; 2}^{\prime}( \pm c, 0)$ is obtained from (5.3) and using Corollary 8.2 of [9].

Let us define the Faddeev function from the left corresponding to $V_{2}$ :

$$
m_{l ; 2}(k, x):=e^{-i \gamma(k) x} f_{l ; 2}(k, x)
$$

Using (5.3), (5.6), and (5.13) we get

$$
X(\gamma)=\frac{m_{l ; 2}^{\prime}(k(\gamma), 0)}{m_{l ; 2}(k(\gamma), 0)}, \quad \gamma \in \mathbf{R} .
$$

From (5.3), (5.13), and (5.14) it is seen that $X(\gamma)$ is well defined for $\gamma \in \mathbf{R} \backslash\{0\}$, it is well defined also at $\gamma=0$ if $L_{2}(c) \neq-1$, and $X(0)=0$ if $L_{2}(c)=1$.

Proposition 5.4 Assume that $V_{2}$ is real valued, it has support in $\mathbf{R}^{+}$, and $V_{2}-c^{2} \in$ $L_{1}^{1}\left(\mathbf{R}^{+}\right)$. Then, for each fixed $x \in \mathbf{R}, m_{l ; 2}(k(\gamma), x)$ and $m_{l ; 2}^{\prime}(k(\gamma), x)$ are continuous in $\gamma \in \overline{\mathbf{C}^{+}}$and analytic in $\gamma \in \mathbf{C}^{+}$. We have

$$
m_{l ; 2}(-k(\gamma), x)=m_{l ; 2}(k(-\gamma), x)=m_{l ; 2}(k(\gamma), x)^{*}, \quad \gamma \in \mathbf{R}
$$




$$
\begin{array}{cl}
m_{l ; 2}^{\prime}(-k(\gamma), x)=m_{l ; 2}^{\prime}(k(-\gamma), x)=m_{l ; 2}^{\prime}(k(\gamma), x)^{*}, & \gamma \in \mathbf{R}, \\
m_{l ; 2}(k(\gamma), x)=1+O(1 / \gamma), \quad m_{l ; 2}^{\prime}(k(\gamma), x)=o(1), & \gamma \rightarrow \infty \text { in } \overline{\mathbf{C}^{+}}
\end{array}
$$

Moreover, $X(-\gamma)=X(\gamma)^{*}$ for $\gamma \in \mathbf{R} \backslash\{0\}$, and hence $\operatorname{Im}\{X(\gamma)\}$ is an odd function of $\gamma \in \mathbf{R} \backslash\{0\}$.

PROOF: Using (2.1), (2.4), and (5.13), one obtains the integral relations

$$
\begin{gathered}
m_{l ; 2}(k(\gamma), x)=1+\frac{1}{2 i \gamma} \int_{x}^{\infty} d y\left[e^{2 i \gamma(y-x)}-1\right]\left[V_{2}(y)-c^{2}\right] m_{l ; 2}(k(\gamma), y) \\
m_{l ; 2}^{\prime}(k(\gamma), x)=-\int_{x}^{\infty} d y e^{2 i \gamma(y-x)}\left[V_{2}(y)-c^{2}\right] m_{l ; 2}(k(\gamma), y) .
\end{gathered}
$$

Using iteration as in $[1,2]$ on (5.18) and (5.19), we obtain (5.17) and the analyticity and continuity properties stated. We get (5.15) and (5.16) with the help of the fact that $\gamma$ appears as $i \gamma$ in (5.18) and (5.19). Finally, using (5.15) and (5.16) in (5.14), we get $X(-\gamma)=X(\gamma)^{*}$ for $\gamma \in \mathbf{R} \backslash\{0\}$.

Proposition 5.5 Assume that $V_{2}$ is real valued, it has support in $\mathbf{R}^{+}$, it is free of bound states, and $V_{2}-c^{2} \in L_{1}^{1}\left(\mathbf{R}^{+}\right)$. Then, the hypotheses of Proposition 5.2 are satisfied with $g(\gamma)=f_{l ; 2}(k(\gamma), 0)$. Furthermore

$$
\left|f_{l ; 2}(k(\gamma), 0)\right|^{2}=\frac{\gamma}{\gamma+\operatorname{Im}\{X(\gamma)\}}, \quad \gamma \in \mathbf{R},
$$

and hence (5.7) holds.

PROOF: All of the hypotheses of Proposition 5.2, except (i), follow from Proposition 5.4. On the other hand, from Proposition 5.3 we see that $g$ cannot vanish in $\gamma \in \overline{\mathbf{C}^{+}}$, except possibly at $\gamma=0$, in which case the multiplicity of the zero at $\gamma=0$ is one.

To prove (5.20), notice that (5.6) can be written as

$$
L_{2}(k(\gamma))=\frac{i[k(\gamma)-\gamma]-X(\gamma)}{i[k(\gamma)+\gamma]+X(\gamma)}, \quad \gamma \in \mathbf{R}
$$


From (2.6), (2.9), (5.3), (5.15), and (5.21) we get

$$
f_{l ; 2}(k(\gamma), 0) f_{l: 2}(k(-\gamma), 0)=\left|\frac{1+L_{2}(k(\gamma))}{T_{2}(k(\gamma))}\right|^{2}=\frac{\gamma}{k(\gamma)}\left[\frac{\left|1+L_{2}(k(\gamma))\right|^{2}}{1-\left|L_{2}(k(\gamma))\right|^{2}}\right],
$$

and using (5.21) in (5.22) we obtain (5.20).

Proposition 5.6 Assume that $V_{2}$ is real valued, it has support in $\mathbf{R}^{+}$, it is free of bound states, and $V_{2}-c^{2} \in L_{1}^{1}\left(\mathbf{R}^{+}\right)$. Then, the hypotheses of Proposition 5.2 are satisfied with $g(\gamma)=\frac{1}{i(\gamma+i)} f_{l ; 2}^{\prime}(k(\gamma), 0)$. Moreover

$$
\left|\frac{1}{i(\gamma+i)} f_{l ; 2}^{\prime}(k(\gamma), 0)\right|^{2}=\frac{\gamma\left[\gamma^{2}+2 \gamma \operatorname{Im}\{X(\gamma)\}+|X(\gamma)|^{2}\right]}{\left(\gamma^{2}+1\right)[\gamma+\operatorname{Im}\{X(\gamma)\}]}, \quad \gamma \in \mathbf{R},
$$

and hence (5.8) holds.

PROOF: The analyticity and continuity hypotheses as well as (ii) of Proposition 5.2 follow from (5.13) and Proposition 5.4. From (5.3) and Proposition 5.3 we get (i) of Proposition 5.2. Next, notice that (5.13) gives us

$$
\frac{f_{l ; 2}^{\prime}(k(\gamma), 0)}{i(\gamma+i)}=m_{l ; 2}(k(\gamma), 0)+\frac{m_{l ; 2}^{\prime}(k(\gamma), 0)+m_{l ; 2}(k(\gamma), 0)}{i(\gamma+i)},
$$

and (5.17) and (5.24) imply (iii) of Proposition 5.2. Finally, using (5.14) and (5.24) we get

$$
\left|\frac{f_{l ; 2}^{\prime}(k(\gamma), 0)}{i(\gamma+i)}\right|^{2}=\left|m_{l ; 2}(k(\gamma), 0)\left(\frac{i \gamma+X(\gamma)}{i(\gamma+i)}\right)\right|^{2}=\frac{\gamma}{\gamma+\operatorname{Im}\{X(\gamma)\}} \frac{|i \gamma+X(\gamma)|^{2}}{\gamma^{2}+1},
$$

which gives us (5.23). Recall that $X(\gamma)$ is not well defined at $\gamma=0$ if $L_{2}( \pm c)=-1$; notice, however, that we do not need to assume $L_{2}( \pm c) \neq-1$, because the left-hand side in (5.23) has a well defined nonzero limit as $\gamma \rightarrow 0$ when $L_{2}( \pm c)=-1$, which can be seen from (5.3). Since $\frac{1}{i(\gamma+i)} f_{l ; 2}^{\prime}(k(\gamma), 0)$ satisfies all hypotheses of Proposition 5.2, it follows that (5.8) holds.

Proposition 5.7 Assume that $V_{2}$ is real valued and $V_{2}-c^{2} \in L_{1}^{1}\left(\mathbf{R}^{+}\right)$. Then, the quantities $B(t)$ and $E(t)$ defined in (5.9) are both $1+o(1 / t)$ as $t \rightarrow+\infty$. Moreover, the integrands in (5.10) and (5.11) are both $o\left(1 / t^{3}\right)$ as $t \rightarrow+\infty$. 
PROOF: From (5.14) and (5.17) we see that $B(t)$ and $E(t)$ are $1+o(1 / t)$ as $t \rightarrow+\infty$. Because $\log (1+z)=z[1+o(1)]$ as $z \rightarrow 0$, it follows that the integrands in (5.10) and (5.11) are $o\left(1 / t^{3}\right)$ as $t \rightarrow+\infty$.

When $V_{2}$ possesses some bound states, the construction of $L_{2}$ and $T_{2}$ for $k \in \overline{\mathbf{C}^{+}}$using $\left\{L_{2}(k), \gamma \in \mathbf{R}\right\}$ can be carried out by making use of a Darboux transformation allowing the removal of bound states. If the bound states of $V_{2}$ correspond to $k=i \kappa_{j}$ for $j=1, \ldots, N$, then by Proposition 4.1, the set $\left\{\kappa_{j}\right\}_{j=1}^{N}$ is uniquely determined by $\left\{L_{2}(k), c<k<+\infty\right\}$. We can remove the bound states by letting

$$
L_{2}^{[0]}(k):=(-1)^{N} L_{2}(k) \prod_{j=1}^{N}\left(\frac{k-i \kappa_{j}}{k+i \kappa_{j}}\right)
$$

which is the reflection coefficient from the left corresponding [21] to a potential $V_{2}^{[0]}$ with support in $\mathbf{R}^{+}$and satisfying $V_{2}^{[0]}-c^{2} \in L_{1}^{1}\left(\mathbf{R}^{+}\right)$. Let $T_{2}^{[0]}(k)$ denote the corresponding transmission coefficient from the left. By using the method given earlier in this section, we can construct $L_{2}^{[0]}(k)$ and $T_{2}^{[0]}(k)$ for $k \in \overline{\mathbf{C}^{+}}$from $\left\{L_{2}^{[0]}(k(\gamma)), \gamma \in \mathbf{R}\right\}$. Then, the bound states can be restored by letting [21]

$$
T_{2}(k)=T_{2}^{[0]}(k) \prod_{j=1}^{N}\left(\frac{\gamma(k)+i \sqrt{\kappa_{j}^{2}+c^{2}}}{k-i \kappa_{j}}\right), \quad L_{2}(k)=(-1)^{N} L_{2}^{[0]}(k) \prod_{j=1}^{N}\left(\frac{k+i \kappa_{j}}{k-i \kappa_{j}}\right)
$$

\section{EXAMPLES}

In this section we illustrate the theory presented earlier with three examples. Our first example below shows that there may exist two distinct potentials corresponding to the same data (3.1), so that no further improvement in Theorem 3.1 is possible, in general.

Example 6.1 Let $c=0$ and

$$
V_{1}(x)=-2 H(-x) \frac{d^{2}}{d x^{2}} \log \left(1+\frac{1}{6}(\sqrt{13}-2)(\sqrt{13}+3) e^{2 \sqrt{13} x}\right) H(-x),
$$




$$
\begin{gathered}
V_{2}(x)=-2 H(x) \frac{d^{2}}{d x^{2}} \log \left(1+\frac{1}{3}(\sqrt{10}+1)(\sqrt{10}-3) e^{-2 \sqrt{10} x}\right) \\
\tilde{V}_{2}(x)=-2 H(x) \frac{d^{2}}{d x^{2}} \log \left(1+\frac{e^{-4 x}}{18}(\sqrt{10}+2)^{2}+\frac{e^{-2 \sqrt{10} x}\left[(\sqrt{10}+2)^{2}+2 e^{-4 x}\right]}{2(\sqrt{10}+1)(\sqrt{10}+3)}\right),
\end{gathered}
$$

where we recall that $H(x)$ is the Heaviside function. The corresponding scattering coefficients are given by

$$
\begin{array}{cc}
R_{1}(k)=-\frac{6}{(k+2 i)(k-3 i)}, & T_{1}(k)=\frac{k(k+\sqrt{13} i)}{(k+2 i)(k-3 i)}, \\
L_{2}(k)=-\frac{3}{(k-i)(k+3 i)}, & T_{2}(k)=\frac{k(k+\sqrt{10} i)}{(k-i)(k+3 i)}, \\
\tilde{L}_{2}(k)=-\frac{3(k+2 i)}{(k+i)(k-2 i)(k+3 i)}, & \tilde{T}_{2}(k)=\frac{k(k+2 i)(k+\sqrt{10} i)}{(k+i)(k-2 i)(k+3 i)} .
\end{array}
$$

From the poles in $\mathbf{C}^{+}$of the transmission coefficients given in (6.1)-(6.3) it follows that $V_{1}, V_{2}$, and $\tilde{V}_{2}$ each has one bound state. With the help of (4.6) it can be shown that $V:=V_{1}+V_{2}$ also has one bound state, but $\tilde{V}:=V_{1}+\tilde{V}_{2}$ has two. It is easily seen that $\left|L_{2}\right| \equiv\left|\tilde{L}_{2}\right|$ and $\left|T_{2}\right| \equiv\left|\tilde{T}_{2}\right|$. We also have $R_{1}^{*} L_{2}^{*}=R_{1} \tilde{L}_{2}$ so that

$$
|T(k)|=\frac{\left|T_{1}(k) T_{2}(k)\right|}{\left|1-R_{1}(k) L_{2}(k)\right|}=\frac{\left|T_{1}(k) \tilde{T}_{2}(k)\right|}{\left|1-R_{1}^{*}(k) L_{2}^{*}(k)\right|}=\frac{\left|T_{1}(k) \tilde{T}_{2}(k)\right|}{\left|1-R_{1}(k) \tilde{L}_{2}(k)\right|}=|\tilde{T}(k)| .
$$

Consequently $|L| \equiv|\tilde{L}|$, and so $V_{2}$ and $\tilde{V}_{2}$ cannot be distinguished by the measurement data (3.1). Note that we could also get $L$ and $\tilde{L}$ explicitly by using [10]

$$
L(k)=\left[\frac{L_{2}(k)-R_{1}(k)^{*}}{1-R_{1}(k) L_{2}(k)}\right] \frac{T_{1}(k)}{T_{1}(k)^{*}}, \quad k \in \mathbf{R},
$$

and a similar formula for $\tilde{L}$ and verify that $|L| \equiv|\tilde{L}|$.

Next, we illustrate the reconstruction of $V_{2}$ from the data (3.1) when there are no bound states and all the potentials involved are generic. 
Example 6.2 Assume $c=1$ and that neither $V_{1}$ nor $V_{2}$ has any bound states. Let us use the data $\left\{R_{1},\left|L_{2}\right|,|L|\right\}$, where $\gamma=\sqrt{k^{2}-1}$ and

$$
\begin{gathered}
R_{1}(k)=\frac{2}{2 k^{2}+3 i k-2}, \quad k \in(0,+\infty), \\
\left|L_{2}\right|^{2}=\left\{\begin{array}{l}
1, \quad k \in[0,1], \\
\frac{2 k^{4}-2 k^{3} \gamma-k^{2}+12}{2 k^{4}+2 k^{3} \gamma-k^{2}+12},
\end{array}\right. \\
|L|^{2}=\left\{\begin{array}{l}
1, \quad k \in[1,+\infty), \\
\frac{8 k^{8}-8 k^{7} \gamma-2 k^{6}-2 k^{5} \gamma+7 k^{4}+300 k^{2}}{8 k^{8}+8 k^{7} \gamma-2 k^{6}+2 k^{5} \gamma+7 k^{4}+300 k^{2}},
\end{array} \quad k \in(1,+\infty) .\right.
\end{gathered}
$$

We are interested in constructing $V_{2}$. For $k \in(1,+\infty)$, from the scattering data we get

$$
\begin{gathered}
1-\left|R_{1}\right|^{2}=\frac{k^{2}\left(4 k^{2}+1\right)}{4 k^{4}+k^{2}+4}, \\
1-\left|L_{2}\right|^{2}=\frac{4 k^{3} \gamma}{2 k^{4}+2 k^{3} \gamma-k^{2}+12}, \\
1-|L|^{2}=\frac{4 k^{5}\left(4 k^{2}+1\right) \gamma}{8 k^{8}+8 k^{7} \gamma-2 k^{6}+2 k^{5} \gamma+7 k^{4}+300 k^{2}}
\end{gathered}
$$

Next, using (4.7) and (5.2) we evaluate $A(k)$ and $Z(k)$ for $k \in(1,+\infty)$; we obtain

$$
\begin{gathered}
A=\frac{28 k^{4}-148 k^{2}+48}{8 k^{8}+8 k^{7} \gamma-2 k^{6}+2 k^{5} \gamma+55 k^{4}+8 k^{3} \gamma+8 k^{2}+48}, \\
Z=\frac{32400 k^{6}-86400 k^{4}+57600 k^{2}}{\left(4 k^{4}+k^{2}+4\right)^{2}\left(2 k^{4}+2 k^{3} \gamma-k^{2}+12\right)^{2}}
\end{gathered}
$$

or equivalently

$$
Z=\frac{3600 k^{2}\left(3 k^{2}-4\right)^{2}}{\left(4 k^{4}+k^{2}+4\right)^{2}\left(2 k^{4}+2 k^{3} \gamma-k^{2}+12\right)^{2}} .
$$

Hence, in the interval $k \in[1,+\infty)$, the quantity $\sqrt{Z(k)}$ has a simple zero at $k=2 / \sqrt{3}$. Here we use the branch of the (complex) square-root function such that the analytic continuation of $\sqrt{Z(k)}$ remains nonnegative as $k \rightarrow 0^{+}$, i.e. we get

$$
\sqrt{Z(k)}=\frac{60 k\left(4-3 k^{2}\right)}{\left(4 k^{4}+k^{2}+4\right)\left(2 k^{4}+2 k^{3} \gamma-k^{2}+12\right)} .
$$


Thus, from (5.1) we obtain

$$
\begin{gathered}
\operatorname{Re}\{F(k)\}=\frac{8 k^{8}+8 k^{7} \gamma-2 k^{6}+2 k^{5} \gamma+27 k^{4}+8 k^{3} \gamma+156 k^{2}}{8 k^{8}+8 k^{7} \gamma-2 k^{6}+2 k^{5} \gamma+55 k^{4}+8 k^{3} \gamma+8 k^{2}+48}, \\
\operatorname{Im}\{F(k)\}=\frac{30 k\left(3 k^{2}-4\right)}{\left(4 k^{4}+k^{2}+4\right)\left(2 k^{4}+2 k^{3} \gamma-k^{2}+12\right)} .
\end{gathered}
$$

Note that since there are no bound states and $V_{1}, V_{2}$, and $V$ are all generic, we have used $\operatorname{Im}\{F(k)\}=-\frac{1}{2} \sqrt{Z(k)}$, as indicated in [10]. Thus, we have constructed $F(k)$ as

$$
F(k)=\frac{\left(8 k^{8}+8 k^{7} \gamma-2 k^{6}+2 k^{5} \gamma+27 k^{4}+8 k^{3} \gamma+156 k^{2}\right)+30 i k\left(3 k^{2}-4\right)}{8 k^{8}+8 k^{7} \gamma-2 k^{6}+2 k^{5} \gamma+55 k^{4}+8 k^{3} \gamma+8 k^{2}+48} .
$$

Although the expression in (6.4) has been constructed for $k \in(1,+\infty)$, by analytic continuation it is valid also for $k \in[0,1]$. We have

$$
1-F=\frac{\left(28 k^{4}-148 k^{2}+48\right)-30 i k\left(3 k^{2}-4\right)}{8 k^{8}+8 k^{7} \gamma-2 k^{6}+2 k^{5} \gamma+55 k^{4}+8 k^{3} \gamma+8 k^{2}+48},
$$

or equivalently

$$
1-F=\frac{\left(28 k^{4}-148 k^{2}+48\right)-30 i k\left(3 k^{2}-4\right)}{\left(4 k^{4}+k^{2}+4\right)\left(2 k^{4}+2 k^{3} \gamma-k^{2}+12\right)}
$$

Next, using (4.8) and (6.5), we get

$$
\frac{1-F(k)}{R_{1}(k)}=\frac{28 k^{4}-90 i k^{3}-148 k^{2}+120 i k+48}{\left(4 k^{2}-6 i k-4\right)\left(2 k^{4}+2 k^{3} \gamma-k^{2}+12\right)}
$$

and thus, after some simplification, we obtain

$$
L_{2}(k)=\frac{1-F(k)}{R_{1}(k)}=\frac{7 k^{2}-12 i k-12}{2 k^{4}+2 k^{3} \gamma-k^{2}+12}, \quad k \in \mathbf{R},
$$

which corresponds to the potential given by

$$
V_{2}(x)=\left[1+\frac{24 e^{2 x}}{\left(3 e^{2 x}-1\right)^{2}}\right] H(x)
$$

In our third example, we illustrate the construction of $\left\{T_{2}(k), k \in \overline{\mathbf{C}^{+}}\right\}$corresponding to $\left\{L_{2}(k), c<k<+\infty\right\}$ studied in Example 6.2. 
Example 6.3 Assume $c=1$ and

$$
L_{2}(k)=\frac{7 k^{2}-12 i k-12}{2 k^{4}+2 k^{3} \gamma-k^{2}+12}, \quad k \in(1,+\infty)
$$

We are interested in finding $T_{2}$ for $k \in \overline{\mathbf{C}^{+}}$. Using (6.6) in (5.6) we get

$$
X(\gamma)=\frac{-3 i k^{2}-3 i k \gamma-6 k-6 \gamma+12 i}{k^{3}+k^{2} \gamma+3 k-6 i}, \quad \gamma \in \mathbf{R}
$$

which simplifies to $X(\gamma)=-3 i /(\gamma+2 i)$. Then, after some simplification we see that $(5.20)$ can be written as

$$
f_{l ; 2}(k(\gamma), 0) f_{l ; 2}(k(-\gamma), 0)=\frac{(\gamma+2 i)(\gamma-2 i)}{(\gamma+i)(\gamma-i)}, \quad \gamma \in \mathbf{R}
$$

from which we get

$$
f_{l ; 2}(k(\gamma), 0)=\frac{\gamma+2 i}{\gamma+i}, \quad \gamma \in \mathbf{R}
$$

Using (5.5), (5.14), and (6.7), after some simplification, we obtain

$$
T_{2}(k)=\frac{2 k(\gamma+i)}{k^{2}+k \gamma-4+2 i(k+\gamma)} .
$$

Note that (6.8) holds for $k \in \overline{\mathbf{C}^{+}}$.

In our fourth example, we show that even an infinite set of reflectivity measurements with all distinct reference potentials may provide no more information than what the two-measurement data (3.1) provide.

Example 6.4 Consider the one-parameter family of reflection coefficients

$$
R_{1}(k ; C)=\frac{C}{(k+i)(k+2 i)}, \quad C \in(-2,2]
$$

The potentials $V_{1}(x ; C)$ corresponding to $R_{1}(k ; C)$ do not contain any bound states, are supported in $\mathbf{R}^{-}$, and are given by

$$
V_{1}(x ; C)=-2 H(-x) \frac{d^{2}}{d x^{2}} \log \left(1-a_{+} e^{2 \epsilon_{+} x}-a_{-} e^{2 \epsilon_{-} x}+a_{+} a_{-}\left(\frac{\epsilon_{+}-\epsilon_{-}}{\epsilon_{+}+\epsilon_{-}}\right)^{2} e^{2\left(\epsilon_{+}+\epsilon_{-}\right) x}\right)
$$


where

$$
\epsilon_{ \pm}=\sqrt{\frac{5 \pm \sqrt{4 C^{2}+9}}{2}}, \quad a_{ \pm}= \pm \frac{C\left(\epsilon_{+}+\epsilon_{-}\right)}{\left(\epsilon_{ \pm}+1\right)\left(\epsilon_{ \pm}+2\right)\left(\epsilon_{+}-\epsilon_{-}\right)} .
$$

Note that $V_{1}(x ; C)$ is generic for $C=2$ and exceptional for $C \in(-2,2)$, and it decays exponentially as $x \rightarrow-\infty$. For example, when $C=2$ we get

$$
V_{1}(x ; 2)=\frac{80(\sqrt{5}+1)(\sqrt{5}+2) e^{-2 \sqrt{5} x}}{\left[(\sqrt{5}+1)(\sqrt{5}+2) e^{-2 \sqrt{5} x}-2\right]^{2}} H(-x) .
$$

Any two members in the family given in (6.9) have either identical phases for all $k \in \mathbf{R}$ or their phases differ by $\pi$ for all $k \in \mathbf{R}$; in other words, $R_{1}(k ; C) R_{1}(k ; \tilde{C})^{*}$ is real for any $C, \tilde{C} \in(-2,2]$ and for all real $k$. Hence, according to Corollary 3.2 of [10], the reflectivity data collected by using any two potentials in this family (one of these can be chosen as $C=$ $0)$ contain all the information towards the determination of $L_{2}$. No extra measurements by using any potentials in this family can give us any additional information.

In our final example we demonstrate a numerical implementation of the analytic continuation quadrature given in Section 5 .

Example 6.5 Consider the potential $V_{2}$ shown in Figure 1 , where $V_{2} \equiv 0$ for $x<0$ and $V_{2} \equiv 1$ for $x>1$. We are able to obtain $\left\{L_{2}(k), 0<k<+\infty\right\}$ numerically to any desired accuracy via a numerical solution of (2.1). In our example, we have solved the direct problem for $0<k<20$ on a grid of spacing $\Delta k=.01$ and have obtained the 'exact' $L_{2}(k)$ for $0<k<20$. Now assuming that we know the 'exact' $L_{2}(k)$ only for $1<k<+\infty$, we compute $L_{2}(k)$ for $0<k<1$ by using the quadrature described in Section 5 . We use Matlab for the numerical implementation and proceed as follows:

(i) We are given $L_{2}(k)$ for $c<k<k_{\max }$ at the discrete points $k_{j}$, where $c=1, k_{\max }=20$, and our grid spacing is $\Delta k=0.01$.

(ii) We compute $X(\gamma)$ from (5.6) for $0<\gamma<\gamma_{\max }$ at the discrete points $\gamma_{j}$, where $\gamma_{j}:=\sqrt{k_{j}^{2}-c^{2}}$ and $\gamma_{\max }:=\sqrt{k_{\max }^{2}-c^{2}}$. 
(iii) Next we compute $B(\gamma)$ and $E(\gamma)$ on the $\gamma$ grid by using (5.9).

(iv) Now for any fixed $k$ of interest in $(0, c)$, and hence $\gamma=i \sqrt{c^{2}-k^{2}}$, by replacing the upper endpoint $+\infty$ with $\gamma_{\max }$ we evaluate the integrals in (5.10) and (5.11) with the Matlab 'quad8' function; since this requires the integrand to be calculable at any point, we use Matlab 'interp1' for the spline interpolation of $B$ and $E$. Then, from (5.4) we compute $L_{2}(k)$ for $0<k<c$.

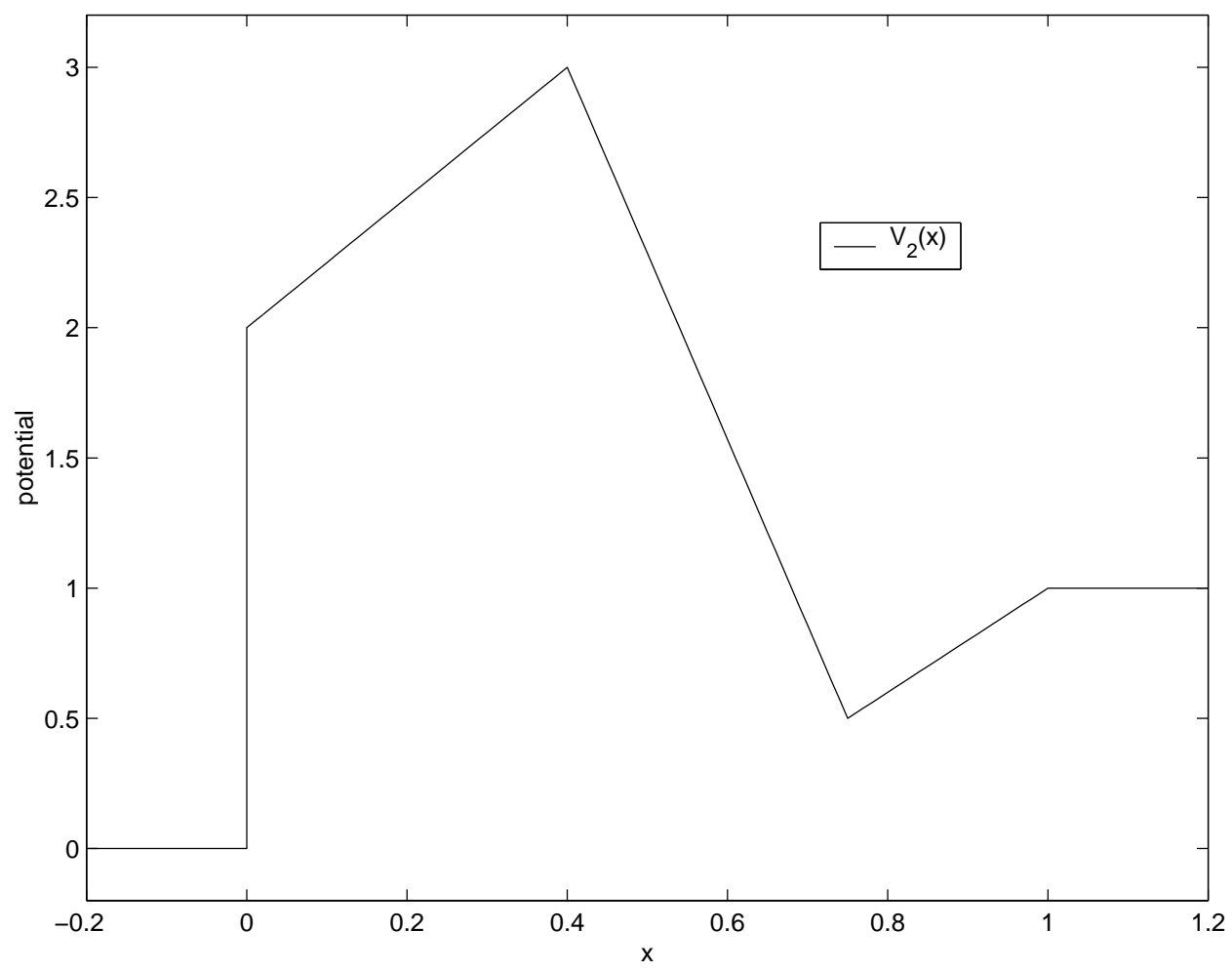

Figure 1 The potential $V_{2}$ used in Example 6.5

In Figure 2 we have graphed the phase of $L_{2}(k)$ for $0<k<c$, showing both the exact and computed values. Our analytic continuation quadrature described in Section 5 seems to be easy to implement numerically and in general such an implementation results in good agreement between the exact and computed phases. Since we are evaluating (5.10) and (5.11) when $\gamma$ is purely imaginary, the integrands are in fact nonsingular. Moreover, as indicated in Proposition 5.7, the integrands decay as $o\left(1 / t^{3}\right)$ as $t \rightarrow+\infty$, and hence replacing the upper limit $+\infty$ by $\gamma_{\max }$ in the numerical evaluation in step (iv) is justified. 
The only expected instability may occur if we let $-i \gamma \rightarrow 0^{+}$, i.e. when $k \rightarrow c^{-}$, in step (iv).

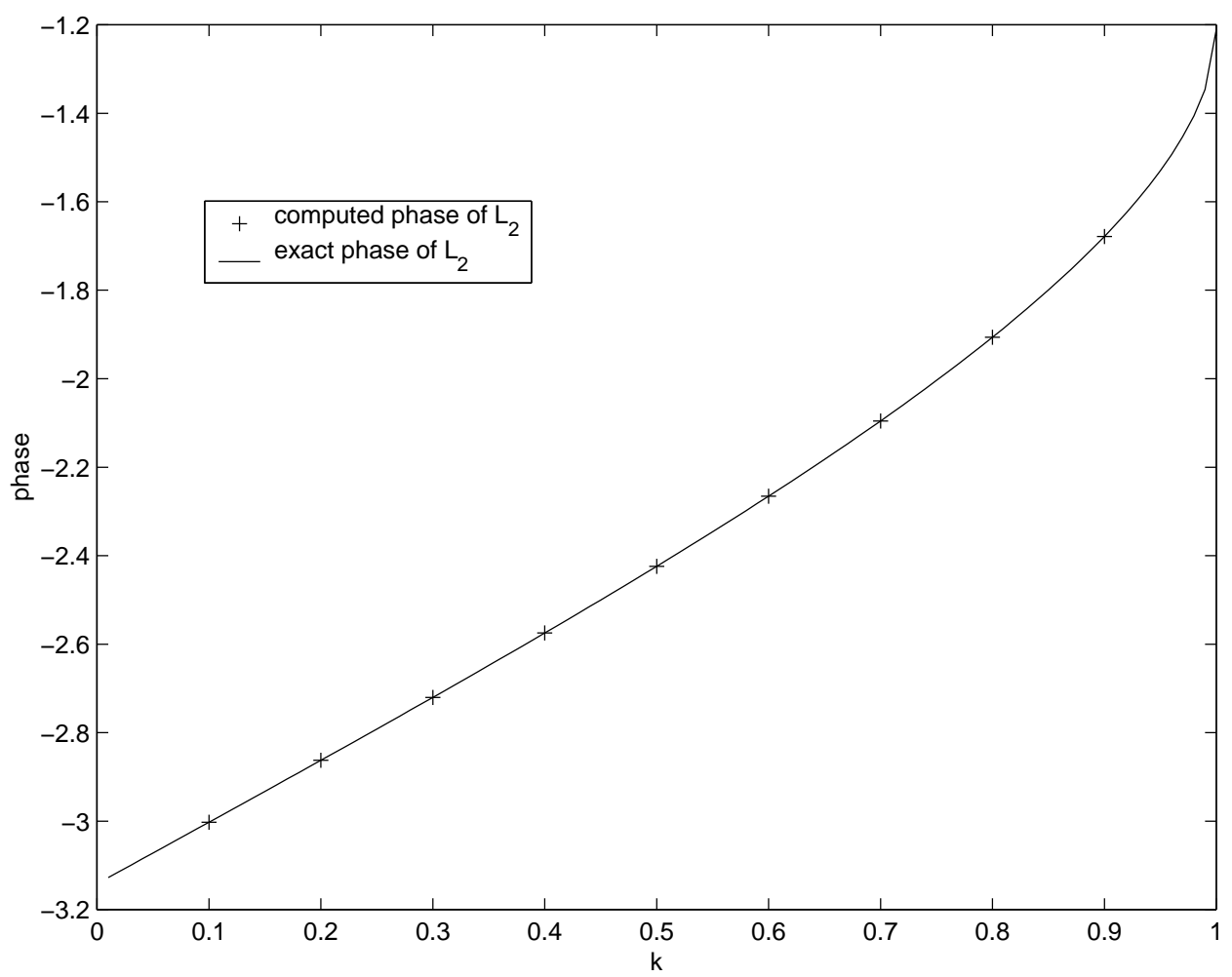

Figure 2 The exact phase and the computed phase of $L_{2}$ for $k \in(0,1)$ in Example 6.5

Acknowledgments. The research leading to this article was supported in part by the National Science Foundation under grants DMS-9803219 and DMS-9504611.

\section{REFERENCES}

[1] L. D. Faddeev, Properties of the S-matrix of the one-dimensional Schrödinger equation, Amer. Math. Soc. Transl. (Ser. 2) 65, 139-166 (1967) [Trudy Mat. Inst. Steklova 73, 314-336 (1964) (Russian)].

[2] P. Deift and E. Trubowitz, Inverse scattering on the line, Comm. Pure Appl. Math. 32, 121-251 (1979).

[3] K. Chadan and P. C. Sabatier, Inverse problems in quantum scattering theory, 2nd 
ed., Springer, New York, 1989.

[4] C. F. Majkrzak and N. F. Berk, Exact determination of the phase in neutron reflectometry, Phys. Rev. B 52, 10827-10830 (1995).

[5] V. O. de Haan, A. A. van Well, S. Adenwalla, and G. P. Felcher, Retrieval of phase information in neutron reflectometry, Phys. Rev. B 52, 10831-10833 (1995).

[6] T. Aktosun and P. Sacks, Inverse problem on the line without phase information, Inverse Problems 14, 211-224 (1998).

[7] C. F. Majkrzak and N. F. Berk, Exact determination of the phase in neutron reflectometry by variation of the surrounding medium, Phys. Rev. B 58, 15416-15418 (1998).

[8] A. Cohen and T. Kappeler, Scattering and inverse scattering for steplike potentials in the Schrödinger equation, Indiana Univ. Math. J. 34, 127-180 (1985).

[9] T. Aktosun, On the Schrödinger equation with steplike potentials, J. Math. Phys., to appear (1999) [the manuscript is available from the author's home page at the url http://www.math.ndsu.nodak.edu/faculty/aktosun/preprints.html].

[10] T. Aktosun and P. Sacks, Inversion of reflectivity data for nondecaying potentials, SIAM J. Appl. Math., to appear [available from the first author's home page at the url http://www.math.ndsu.nodak.edu/faculty/aktosun/preprints.html].

[11] V Buslaev and V. Fomin, An inverse scattering problem for the one-dimensional Schrödinger equation on the entire axis, Vestnik Leningrad. Univ. 17, 56-64 (1962) (Russian).

[12] J. Legendre, Problème inverse de Schrödinger sur la ligne avec conditions dissymétri ques et applications, Ph.D. thesis, Acad. Montpellier, Univ. Sci. Tech. Languedoc, 1982. 
[13] V. A. Marchenko, Sturm-Liouville operators and applications, Birkhäuser, Basel, 1986.

[14] M. Klaus, Low-energy behaviour of the scattering matrix for the Schrödinger equation on the line, Inverse Probl. 4, 505-512 (1988).

[15] V. O. de Haan, A. A. van Well, P. E. Sacks, S. Adenwalla, and G. P. Felcher, Toward the solution of the inverse problem in neutron reflectometry, Phys. B 221, 524-532 (1996).

[16] C. F. Majkrzak and N. F. Berk, Exact determination of the neutron reflection amplitude or phase, Phys. B 221, 520-523 (1996).

[17] C. F. Majkrzak, N. F. Berk, J. Dura, S. K. Satija, A. Karim, J. Pedulla, and R. D. Deslattes, Direct inversion of specular reflectometry, Phys. B. 241-243, 1101-1103 (1998)

[18] N. N. Novikova and V. M. Markushevich, Uniqueness of the solution of the onedimensional problem of scattering for potentials located on the positive semiaxis, Comput. Seismology 18, 164-172 (1987) [Vychislitel'naya Seismologiya 18, 176-184 (1985) (Russian)].

[19] T. Aktosun, Bound states and inverse scattering for the Schrödinger equation in one dimension, J. Math. Phys. 35, 6231-6236 (1994).

[20] H. Dym and H. P. McKean, Gaussian processes, function theory, and the inverse spectral problem, Academic Press, New York, 1976.

[21] T. Aktosun, Darboux transformation for the Schrödinger equation with steplike potentials, preprint (1999) [the manuscript is available from the author's home page at the url http://www.math.ndsu.nodak.edu/faculty/aktosun/preprints.html]. 